\title{
Microemprendimientos en agricultura ecológica y mercado slow food
}

\author{
Rocío Blanco-Gregory \\ Universidad de Extremadura \\ rblanco@unex.es \\ M. ${ }^{a}$ Violante Martínez-Quintana \\ Universidad Nacional de Educación a Distancia \\ vmartin@poli.uned.es \\ M. ${ }^{a}$ Victoria Sanagustín-Forns \\ Universidad de Zaragoza \\ vitico@unizar.es
}

Resumen: En este artículo se presenta una investigación de carácter cualitativo que se ha llevado a cabo sobre una amplia muestra de microemprendedores en el estado mexicano de Yucatán; los cuales, motivados por el consumo de productos saludables $y$ de carácter orgánico en el campo de la agroecología, se han iniciado en sus negocios, basados, principalmente, en la producción y comercialización de este tipo de productos de origen ganadero o agrícola, pero fundamentalmente de este último.

En concreto, se quiere exponer, más detalladamente, la opinión que tienen estos microemprendedores agroecológicos sobre los productores de su entorno, es decir, sobre la competencia en el mercado yucateco, indagando, asimismo, en relación con la existencia de redes sociales e interacción social entre los emprendedores, y con las formas de cooperación que existen entre unos y otros.

Palabras clave: microemprendimientos; producción agroecológica; productos orgánicos; redes sociales; slow food. 


\section{Microbusinesses in organic farming and slow food market}

Abstract: This article presents a qualitative investigation that has been carried out on a large sample of microentrepreneurs in the Mexican state of Yucatán; which, motivated by the consumption of healthy and organic products in the field of agroecology, have started in their businesses, based mainly on the production and marketing of this type of products of livestock or agricultural origin, but mainly from the last batch.

Specifically, we want to expose, in more detail, the opinion that these agroecological micro-entrepreneurs have about the producers in their environment, that is, about the competition in the Yucatecan market, also inquiring about the existence of social networks and social interaction among entrepreneurs; and about the forms of cooperation that exist between them.

Keywords: Micro-businesses, agroecological production, organic products, social networks, slow food. 


\section{Introducción y objetivos}

Esta investigación de carácter cualitativo ha sido realizada sobre una amplia muestra de microemprendedores en el estado mexicano de Yucatán (México). Estos emprendedores están motivados tanto por el consumo de productos saludables y de carácter orgánico en el campo de la agroecología como por enfocar sus negocios (la mayoría de ellos en el período de los últimos ocho-diez años) en la producción y comercialización de este tipo de productos de origen ganadero y agrícola.

En concreto, el objeto de estudio de esta investigación es conocer, en profundidad, qué opinión tienen estos microemprendedores agroecológicos sobre los productores de su entorno, es decir, sobre la competencia en el mercado yucateco; asimismo, se plantea indagar sobre la existencia o no de redes sociales y la interacción social entre estos emprendedores; cómo dichas redes impactan en el desarrollo de sus empresas en el sector de los productos ecológicos, analizando su presencia en mercados y ferias, fundamentalmente.

\section{Fundamentación teórica}

\subsection{Microemprendimientos, desarrollo endógeno, sostenible y slow food}

La agricultura orgánica o ecológica existe desde hace más de cien años, pero no es hasta los años noventa del pasado siglo cuando tanto la producción como el consumo se expanden rápidamente, alcanzando tasas de crecimiento por encima del $25 \%$. México es un país que participa en ese movimiento de carácter mundial como productor y exportador de alimentos orgánicos. No obstante, hasta los primeros años del siglo xxi la población mexicana no empieza a conocer y apreciar ese tipo de alimentos, libres de productos químicos o transformaciones peligrosas para la salud humana; es cuando el mercado doméstico comienza a desarrollarse (Gómez Cruz, 2007). Si bien es imprescindible considerar que en el desarrollo de la propia identidad la alimentación posee un importante papel (Pietrykowski, 2004) aunque el descubrimiento del valor de lo propio haya venido desde fuera.

La Unión Europea ocupa el primer puesto en el movimiento orgánico, ya que se configura como el centro de origen moderno de este, acumulando más experiencia histórica al respecto que cualquier otra región en el mundo; además, tiene las tasas de crecimiento más altas y ha declarado el fomento de su producción y consumo como prioridades de sus políticas públicas. La Federación Internacional del Movimiento Agrícola Orgánico (IFOAM) tiene su sede en Alemania, y se esfuerza en cuidar la autenticidad del movimiento y en asegurar la veracidad de lo 
orgánico. En Europa la alimentación y la identidad se están convirtiendo en algo parecido al euro, una única moneda discursiva común a través de la cual se puede debatir sobre la europeidad y las implicaciones de la globalización económica a principios del siglo xxi (Leitch, 2003: 442).

En esta línea surge el interés de la elaboración de productos ecológicos (orgánicos), así como el desarrollo del mercado slow food, que confluyen en la preservación y defensa de la biodiversidad alimentaria y la cultura gastronómica. El eje principal es el placer de la comida y el acto agrícola que, según Petrini (2007), selecciona alimentos de buena calidad, produce productos que respetan los procesos de producción del medio ambiente, las tradiciones locales, y va a favor de una biodiversidad y agricultura justas y sostenibles. De aquí que se alineen en fila las microempresas, el desarrollo endógeno, la sostenibilidad y el emprendimiento en el ámbito local/rural (Sanagustín-Fons, Moseñe y Gómez y Patiño, 2011), que tienen como denominador común un mercado denominado slow food. En efecto, slow food surge de las culturas alimentarias que rodean a las cocinas regionales en Italia (Jones et alii, 2003). Se trata de un movimiento que nació en el año 1986 en Italia, fundamentalmente, a raíz de una protesta que llevaron a cabo un grupo de periodistas el día que se inauguró un restaurante de comida fast food en la plaza de España de Roma, aunque, su origen, como señalan Miele y Murdoch (2002: 7), se estableció en Bra (Piamonte) por un grupo de escritores y chefs. La principal motivación inmediata fue la creciente preocupación sobre el impacto potencial de la comida rápida "americanizada" en las culturas alimentarias locales. Y dando por sentado (Miele y Murdoch, 2002) que la cultura alimentaria, la sostenibilidad ambiental y la economía local convergen en un conjunto interconectado de vínculos en todo el panorama gastronómico.

En aquel momento se proclamó la necesidad de promover la pausa al comer, los productos naturales de proximidad, las recetas locales y el deleite en el sentido del gusto para proteger el patrimonio alimentario de la humanidad. Dado que, en el contexto italiano, los restaurantes tradicionales mantienen una estrecha conexión con los sistemas de producción alimentaria locales, Slow Food argumentó que su protección requiere la promoción general de las culturas alimentarias locales. Así se estableció sobre la base de una estructura local, coordinada por una sede central en Bra (que actualmente emplea a unas cien personas). Inicialmente estas sucursales se establecieron en todas las regiones italianas (y se llamaron condotte), pero pronto comenzaron a extenderse a otros países europeos y luego a otros lugares más lejanos (donde se llaman convivia) (Miele y Murdoch, 2002), como el caso de México.

A finales de los años ochenta, Slow Food comenzó a intervenir en los circuitos de crecimiento de un vigoroso debate nacional sobre la ampliación de la 
aplicación de la nueva legislación uniforme de la Unión Europea en materia de alimentación e inocuidad (Leitch, 2003). Y así, este movimiento internacional slow food se funda oficialmente en París, donde se firma el manifiesto en 1989. Carlo Petrini interviene en el Foro Global sobre Seguridad Alimentaria y Nutrición en Río+20, la Conferencia de las Naciones Unidas sobre el Desarrollo Sostenible (Slow Food, 2015; Marrone, 2011). Promueve la concienciación de proteger la alimentación autóctona basada en la biodiversidad y el respeto al medio ambiente, acceder a los alimentos buenos, limpios y justos producidos ecológicamente, mantener un intercambio equilibrado entre productores $y$ consumidores, y obtener unos beneficios para la salud (Vilaplana, 2011).

La FAO ${ }^{1}$ en 1999, a través de la Comisión del Codex Alimentarius (FAO y OMS, 2011), define la agricultura orgánica como un sistema holístico de gestión que fomenta y mejora la biodiversidad, los ciclos biológicos y la actividad biológica del suelo. El término orgánico denota un proceso y no un producto, y la demanda de productos orgánicos, proveniente, sobre todo, de países desarrollados, ha creado nuevas oportunidades de exportación para el mundo en desarrollo. Ante tal demanda, muchos países en vías de desarrollo respondieron con la producción orgánica y la exportación de esta. Entre ellos se encuentra México, donde el desarrollo de la agricultura orgánica se inició en los años sesenta a través de agentes extranjeros, conectándose con diferentes operadores mexicanos, solicitándoles la producción de determinados productos orgánicos. Y así comenzó su cultivo, fundamentalmente en áreas donde los insumos de síntesis química no eran empleados, como en las regiones indígenas y áreas de agricultura tradicional en los estados de Chiapas y Oaxaca, donde se comenzó con la producción de café orgánico (Schwentesius, 2010), o el maíz, un producto que fue asociado con el campesinado y denigrado por las élites coloniales como nutricionalmente inferior al trigo y que más tarde se convirtió en el centro del desarrollo de una cocina nacional (Leitch, 2003). Más adelante, se fue desarrollando este tipo de cultivos en otros estados del país, como es el caso de Yucatán, sobre el que se contextualiza espacialmente esta investigación.

La agricultura orgánica utiliza métodos naturales de lucha contra las plagas y no de plaguicidas sintéticos, y la fertilidad reciclable de los residuos agrícolas (paja, rastrojos y otras partes no comestibles). Sus objetivos, además de ser económica, tienen como propósito lograr una interacción idónea entre la tierra, los animales y las plantas, conservar los nutrientes naturales y los flujos de energía, y potenciar la diversidad biológica, con miras a conservar los recursos naturales y los ecosistemas para generaciones futuras. No obstante, se han vertido críticas

1 Organización de las Naciones Unidas para la Alimentación y la Agricultura. 
sobre el estado de la cuestión de la agricultura orgánica (Zamilpa, Schwentesius y Ayala, 2016).

Paulatinamente se va produciendo un desplazamiento del actual modelo económico lineal a un modelo cíclico de la naturaleza, donde emergen una economía verde, circular, una movilidad ecointeligente, un desarrollo endógeno, una gestión sostenible, un producto turístico sostenible y una lucha contra el cambio climático (Naciones Unidas, 2012; Junta de Extremadura et alii, 2016; Lupo, 2012; Martínez-Quintana y Blanco-Gregory, 2013; Martínez-Quintana, 2017).

La OCDE, en su informe El nuevo paradigma rural. Politica y gobernanza (OECD, 2006), trata de explicar el cambio de paradigma en las políticas de desarrollo rural emprendido, donde se tienen en cuenta la diversidad de las regiones rurales, los problemas de declive (la emigración, el envejecimiento, el deterioro de las cualificaciones, la disminución de la productividad de la mano de obra), así como el aprovechamiento de las oportunidades y ventajas de los activos de que se dispone (la ubicación, las ventajas naturales y las culturales, y el capital social).

Se trata de un cambio de paradigma rural, desde una nueva visión de la gobernanza, con la participación de los actores del medio rural y de un motor de desarrollo del territorio. Y una multidimensionalidad del desarrollo rural: la endogeneidad, la producción de novedades, la sostenibilidad, el capital social, los nuevos marcos institucionales y la gobernanza de mercados, y culminan sus análisis con una percepción del desarrollo rural, "que puede ser entendido como la mejora de las condiciones económicas, sociales y culturales de un territorio rural, con respeto por el entorno físico y la forma que repercute positivamente sobre la calidad de vida de la población residente e integre el territorio en el conjunto de la sociedad" (Guinjoan et alii, 2016: 197).

Con estas tendencias, el paradigma alternativo de la agricultura ecológica y el paradigma rural se posicionan ante la triple crisis de la actualidad: económica, ambiental y energética, y se vuelve a la cultura del cultivo del huerto (Roiatti, 2011; Ayuntamiento de Madrid, 2017; Merçon et alii, 2012) y a los proyectos de producción agroecológica (Gobierno de España 2009 y 2019), los programas empleaverde, el turismo rural, el patrimonio natural y biodiversidad, las energías limpias y, por último, los retos del futuro (demográficos, psicosociales, económicos, tecnológico-educativos y ambientales), la ruptura del modelo espacial (centro/periferia) y las coordenadas geográficas (Junta de Extremadura et alii, 2016).

El emprendimiento en el ámbito local/rural y la creación de microempresas encuentran una vertiente en el modelo de alimentación lenta que desarrolla, entre otros, la simbiosis de agricultura y turismo. Con puntos débiles y fuertes, representa un gran potencial sin explotar, y el abastecimiento de productos agrícolas locales mejora la comunidad y el atractivo del destino turístico, en el que las mi- 
croempresas locales obtienen apoyo (Murphy, Croes y Chen, 2012; Di Clemente, De Salvo y Hernández, 2011; Donovan Fortín, Bravo y González, 2010; Benito Hernández, 2009; Camacho Vera, Cervantes Escoto, Cesín Vargas y Palacios Rangel, 2019).

En México la agricultura orgánica está dirigida casi exclusivamente a la exportación. Por ello, México está catalogado en el ámbito internacional como productor-exportador orgánico y no como consumidor. No obstante, aunque el grueso de la producción se exporta, hay contadas excepciones con algunos productos orgánicos, como la miel, la carne y los productos lácteos, que se están produciendo en primer lugar para el consumo nacional (Schwentesius, 2010).

Se evidencia la importancia que adquiere el sistema agroalimentario en el recorrido de la comida, desde la tierra al plato, los cambios habidos en los hábitos de lo que se consume (cuánto, cómo, cuándo y dónde), en la preocupación del alejamiento de las dietas tradicionales y la buena salud, y en los problemas alimentarios que tienen que ver con fenómenos sociales relacionados con la abundancia y la globalización estudiados por la sociología de la alimentación (Díaz Méndez y Gómez Benito, 2008), y con el turismo gastronómico que ofrece la cultura local, que identifica el componente experiencial de la comida como producto turístico y como principal atractivo de un destino (Martínez-Quintana, 2019: 175).

\subsubsection{El mercado slow food en el estado mexicano de Yucatán}

En el estado mexicano de Yucatán surge Slow Food Yucatán como una sucursal local de dicho movimiento; tiene como objetivo el crecimiento y el consumo saludables de alimentos cultivados y producidos localmente, y preservar la historia culinaria única de la región. Establecido en 2010 por el Slow Food Yucatán Convivium en México, el Mercado de la Tierra "Mercado Fresco" se celebra todos los miércoles y sábados en la plaza Colón, una pequeña plaza en Mérida, la capital del estado. El mercado ha sido incluido en "Slow Yucatán: desarrollo de un sistema alimentario bueno, limpio y justo, basado en el modelo de sostenibilidad del proyecto internacional Slow Food Movement", financiado por la W. K. Kellogg Foundation (WKKF), uno de cuyos objetivos será utilizar los Mercados de la Tierra de slow food para consolidar las redes de productores locales a través de la comercialización de sus productos.

\subsection{Redes e interacciones sociales, cooperativismo y emprendimiento en lo local/rural}

La presencia de redes e interacciones sociales entre productores, distribuidores y consumidores en el territorio implica la posibilidad de generar entornos de coo- 
peración en los que se consoliden las apuestas de emprendimiento con mayor facilidad e, incluso, con más posibilidades de éxito (Gómez, Sanagustín-Fons y Sáez, 2000). Si bien la cooperación en este tipo de propuestas socioeconómicas podría ser considerada como un valor sociocultural intrínseco al hecho emprendedor (Sanagustín-Fons, 2011) y como algo ideal y básico para alcanzar los objetivos perseguidos en procesos de suma no cero en los que todos ganan. La realidad de las comunidades rurales es compleja y, en ocasiones, paradójica, observándose elevados niveles de individualismo y falta de confianza interpersonal, tal y como señalan Sanagustín-Fons, Lafita-Cortés y Moseñe (2018). Así, en lo que en un principio podrían considerarse como espacios abiertos al encuentro $y$ que facilitan la comunicación interpersonal y social, la realidad demuestra que la cooperación únicamente se promueve cuando existen riesgos que generan incertidumbres y las poblaciones tienden a unirse para salvaguardar su statu quo. Incluso, en entornos vulnerables y en los que se esperan respuestas procedentes de abajo con un compromiso social máximo (Díaz-Muñoz, 2015).

Siguiendo a Gómez Cruz (2005), el nivel de organización puede definirse en criterios como: 1) la cercanía con el productor o proveedor para mantener contacto con la producción orgánica, disminuir costes de transporte y de energía al promover mercados regionales, obtener buenos precios, y mantener los principios ecológicos, etc.; 2) la posibilidad de obtener productos de baja complejidad en la elaboración y transformación, es decir, sin sustancias químicas que adulteren el valor natural de los alimentos y pongan en riesgo la salud del consumidor y de su familia, y 3) tener un punto de abastecimiento permanente, fijo y de información confiable.

En este sentido, el movimiento slow food descrito, para la persecución de sus objetivos, utiliza unas estrategias que van encaminadas, entre otros, al apoyo de la producción local de alimentos conectando a productores y coproductores, educación a los consumidores, protegiendo la biodiversidad, organización eventos relacionados con los alimentos (p. ej,, mercados slow food) y el fomentado del intercambio y la construcción de redes (asociaciones y cooperativas); estrategias todas ellas fundamentales para el desarrollo de los microemprendedores agroecológicos. Como apuntan estudios previos realizados sobre la producción agroecológica (Gómez Cruz, 2005), en México existe potencial para incrementar el consumo de productos orgánicos, y con la meta de despertar el interés y motivar la participación de los actores involucrados en la esfera de la producción, financiación, investigación y del consumo para consolidar un mercado regional que beneficie y fortifique a pequeños productores, campesinos e indígenas.

En el caso de los emprendimientos en lo local/rural, la situación se revela igualmente compleja. Son varias las razones que avalan esta circunstancia y las 
vamos a circunscribir a cuatro dimensiones básicas y significativas desde la perspectiva sociológica: a) las relaciones y el apoyo de las Administraciones públicas a los proyectos de emprendimiento; b) la presencia o ausencia de valores cooperativos entre las poblaciones locales; c) la existencia o las carencias sociocognitivas de la población en relación con los movimientos cooperativistas y lo que pueden implicar para el desarrollo endógeno y sostenible, así como lo que supone el impulso de las asociaciones de productores o de distribuidores; d) finalmente, se trata de una dimensión relevante que es el denominado "miedo al fracaso" presente en ciertas sociedades con valores socioculturales más bien machistas, tradicionales y premodernos. Así, la explicación de dichas dimensiones se describe de la siguiente forma:

a) Las relaciones con las Administraciones públicas se deberían basar en la transparencia y la confianza (Sanagustín-Fons y Brunet-Icart, 2017), proporcionando dichas estructuras públicas el soporte imprescindible para que los microemprendimientos se lleven a cabo; en un estudio llevado de Ramírez et alii (2017) en México se demuestra que cuando el Gobierno apuesta por este tipo de emprendimientos y apoya su financiación y capacitación, generando estructuras de soporte, los resultados, incluso en caso de extrema vulnerabilidad, son altamente positivos y constituyen proyectos de alta rentabilidad social, ayudando a paliar la condición de pobreza aunque no se alcancen las cotas competitivas máximas. Sin embargo, la realidad demuestra que esta circunstancia de apoyo no se da así exactamente, pues existen prioridades políticas diversas y, además, lagunas de gestión que no permiten que se consoliden las estructuras de apoyo al emprendimiento que serían precisas, tales como los espacios de co-working, los microcréditos blandos, la facilidad y exenciones fiscales, entre otras.

b) La ausencia de valores cooperativos entre las poblaciones locales y rurales, siendo los motivos y razones más emocionales que racionales, pero que impiden la orientación comportamental hacia la unión y la consolidación de estructuras cohesionadas que superen la dicotomía individuo-grupo/ sociedad. La razón explicitada por ciertos autores se centra en aspectos que tienen que ver con el control social de dichas comunidades (Viqueira y Palerm, 1954), así como la desconfianza que generan entornos con ciertos niveles de privación económica y la que generan algunos agentes sociales o políticos que promueven el cambio hacia la cooperación.

c) Las carencias sociocognitivas entre la población rural y sus comunidades, en relación con lo que implica el movimiento cooperativista, por un lado, y el asociacionismo, por otro, determinan el grado de impregnación en el territorio de este tipo de iniciativas. 
d) Por último, hacemos referencia al miedo al fracaso presente en cualquier proyecto de emprendimiento.

El liderazgo empresarial femenino responde con eficacia a las exigencias de entornos competitivos y exigentes en los que se exigen constancia, esfuerzo, cierto grado de pragmatismo y, principalmente, unión (Puyal y Sanagustín-Fons, 2006), si bien el ideal del liderazgo emprendedor debería ser contingente a las situaciones y entornos socioeconómicos, es una realidad que las mujeres responden más y mejor, tal y como se ha expuesto y demuestran estudios recientes orientados a la institucionalización del emprendimiento femenino (Montaño y Pérez, 2015).

\section{Metodología}

En el diseño de esta investigación, se llevaron a cabo entrevistas semiestructuradas a emprendedores yucatecos. Los resultados fueron procesados a través de un análisis de contenido de los textos obtenidos en la transcripción de las entrevistas realizadas (codificación, categorización, tematización); el estudio de dichos textos fue de carácter hermenéutico en un esfuerzo interpretativo-comprensivo de estos (Cárcamo, 2005); colocándonos en una situación diferente respecto a la realidad social observada, considerando significaciones latentes y subjetividades expresadas libremente; asimismo, se llevó a cabo una cuantificación de las respuestas para la observación de ciertas tendencias sociales dentro de dicho grupo de emprendedores. La importancia de la investigación cualitativa radica en la peculiaridad y el valor de los discursos de los informantes, así como de la manifestación libre y espontánea de sus opiniones, que provoca una inducción metodológica enriquecedora del tema investigado.

La muestra escogida es de carácter aleatorio y por conveniencia, seleccionándose como entrevistados a aquellos microemprendedores que acudían semanalmente a exponer y vender sus productos orgánicos en el Mercado slow food de la capital de Yucatán (Mérida) y que manifestaron su disposición a colaborar en la investigación. Dicha muestra está formada por veinte personas y se distribuyó de la siguiente forma: nueve fueron hombres y once, mujeres; considerando que la mayoría de los emprendimientos son liderados precisamente por mujeres. La zona geográfica donde se llevó a cabo la investigación fue el Estado de Yucatán, concretamente, en Mérida (la capital) y otros municipios próximos. Las entrevistas se realizaron entre marzo y abril de 2017 de forma personalizada, siendo grabadas en soporte de audio y tomando notas tanto de las respuestas como de aquellos aspectos significativos para la investigación. 
El guion de las entrevistas se focalizó en conocer la opinión que tienen los microemprendedores agroecológicos sobre el conjunto de los productores de orgánicos de su entorno con el objetivo de profundizar en el grado de competencia y desarrollo que existe en el mercado de este sector. Se abordaron áreas temáticas en torno a las iniciativas de emprendimiento en la producción local agroecológica de los principales municipios del estado de Yucatán. Para ello, se trataron cuestiones relativas a aspectos como los siguientes:

- Conocimiento de los microemprendedores sobre la existencia de redes sociales y grado de interacción social que existe entre ellos en el sector de la producción agroecológica.

- Nivel de conocimiento que tienen sobre asociaciones y cooperativas que funcionen para interrelacionarse en dicho sector.

- Opinión de los microemprendedores sobre los principales motivos que se dan en el conjunto de estos emprendedores del sector agroecológico para interrelacionarse entre ellos.

- Grado de conocimiento que poseen sobre experiencias de emprendimiento agroecológico fracasadas y explicación de los motivos del fracaso.

- Opinión de los microemprendedores de este sector sobre las principales razones imperantes en el colectivo para emprender en negocios de carácter agroecológicos.

- Opinión sobre el sector predominante en la producción y comercialización agroecológicas.

- El conjunto de variables que se han considerado relevantes para la investigación, y con base en las cuales se ha obtenido la muestra de microemprendedores, son las siguientes:

Tabla 1. Selección de variables de la investigación

\begin{tabular}{l} 
VARIABLES UTILIZADAS EN EL ESTUDIO \\
Año de creación de la microempresa \\
Constitución legal de la microempresa \\
Ubicación (municipio) \\
Actividad principal de la microempresa \\
Género del microemprendedor \\
Principales productos que produce y/o comercializa \\
Número de empleados de la microempresa \\
Cifra, en pesos mexicanos, de ventas semanales de la microempresa \\
\hline
\end{tabular}

Fuente: elaboración propia. 
Las principales características de los emprendedores que han sido entrevistados se resumen en el anexo I.

\section{Principales resultados}

\subsection{Opinión de los emprendedores agroecológicos sobre los productores orgánicos del entorno}

\subsubsection{Redes sociales e interacción social entre los emprendedores}

Una gran parte de los informantes que han sido entrevistados creen que sí existe una red social en la cual los productores y/o vendedores de los productos orgánicos interactúan, $70 \%$ frente a un 30\% que opina lo contrario. Sin embargo, a pesar de que una gran mayoría de los entrevistados es consciente de que existe una red social para interactuar, un $40 \%$ de ellos opina que el grado de integración entre los productores/comercializadores es fundamentalmente bajo y un 35\% de ellos opina que es medio. Y así lo ilustran algunas de sus declaraciones proporcionadas en las entrevistas: E4: "Hay muchas envidias y celos"; E8: "Es que hay mucho egoísmo en el mexicano y cada cual tira por sí solo"; E10: "Yo por eso pienso que no tienen éxito acá, porque hay un problema de solidaridad y de integración. Eso es muy común aquí, el competir y apuñalarse, ¡idesde la época de los henequeneros! Está en el ADN del yucateco".

A raíz de estas manifestaciones, se obtiene que la falta de confianza en las redes sociales va minando poco a poco las iniciativas de emprendimiento $y$, por tanto, dificulta considerablemente el hecho de crear empresas de este tipo en el estado de Yucatán, no siendo así en otros estados del país con una idiosincrasia más proclive a la cooperación y ayuda mutua, muy distinta a la de los empresarios yucatecos. Estos resultados confirman algunas teorías consideradas en la fundamentación teórica de este artículo relativas a la interacción social y el establecimiento de redes sociales entre los microemprendedores para el cooperativismo, donde ya algunos autores (Sanagustín-Fons et alii, 2018) aluden a la complejidad y paradoja de las comunidades rurales, en las que se suelen dar altos niveles de individualismo y se denota una falta de confianza interpersonal entre aquellos.

\subsubsection{Conocimiento de asociaciones y cooperativas para interrelacionarse en el sector}

En relación con las asociaciones de productores y/o comercializadores agroecológicos, el desconocimiento aumenta entre los microemprendedores que nos informan, pues a veces se confunden con las cooperativas o, simplemente, no se tiene esa información. El desconocimiento de asociaciones y cooperativas para la pro- 
ducción genera un desconocimiento también de las finalidades de estas organizaciones, como pueden ser las siguientes: aumentar la confianza en el consumidor sobre el conocimiento del distribuidor acerca del productor y del producto orgánico, el cumplimiento de los procesos de producción y transformación agrícola y pecuaria, y encontrar alimentos muy elaborados y de fácil preparación; asegurar un flujo de información relacionada con temas actuales y de interés relacionados la producción y la transformación, y apoyar la decisión de compra del consumidor con conocimiento claro y definido y evitar la confusión al encontrar en el mercado alimentos con diferentes sustantivos como "natural", "artesano", "de la tierra", "tradicional", "integrado", "probióticos", "alimentos funcionales", sin respaldo de una normatividad más allá del etiquetado (Gómez Cruz, 2005). (E10: "Aquí en Yucatán no conozco. Es distinto en Chiapas, donde el café y el chocolate son orgánicos certificados. Aquí no tienen la mayoría certificados". E14: "Sí, Slow Food, Colectivo Milpa, Tierra Madre... No, creo que estas dos últimas son cooperativas"). Quizá esta falta de unión y de interacción en la red social venga propiciada también por el escaso conocimiento de los informantes sobre la existencia de cooperativas o asociaciones para la producción o la comercialización de orgánicos, pues cuando les preguntamos cuántas cooperativas conocen para tales fines, las cifras más altas se sitúan entre quienes no conocen ninguna y entre quienes conocen solo de dos a cuatro cooperativas, siendo estas proporciones más bajas aún cuando se les pregunta por las asociaciones destinadas para tal fin.

\subsubsection{Principales motivos de los emprendedores para interrelacionarse}

A la hora de averiguar los motivos por los que los productores y/o comercializadores se relacionan principalmente - a pesar de que la mayoría indica que el nivel de interrelación es bajo-, las razones principales que aluden son las siguientes: para la comercialización de sus productos (45\%) y para conseguir la expansión de sus negocios (30\%), quedando en tercer y cuarto lugar los motivos de para procurarse suministros y para la producción y generar nuevos productos. Así lo confirma uno de los entrevistados, E1: "Lo que pasa es que, si se juntaran y trabajaran en red y sin envidias, podrían pasarse suministros y generar nuevos productos, porque en vez de crecer, uno produce algo nuevo, el otro lo copia y más barato; me quedo con la primera“.

A la luz de las principales motivaciones aludidas por algunos de los entrevistados, obtenemos que la venta y comercialización de los productos es el principal interés de los microemprendedores de orgánicos en general, denotándose una vez más las tendencias individualistas y los intereses propios por encima de un movimiento comunitario, pues el objetivo de los microemprendedores es que sus 
negocios tengan éxito y no tanto la cooperación para que las iniciativas se desarrollen y ayuden a la consolidación del movimiento slow food entre la población yucateca.

\subsubsection{Conocimiento sobre experiencias de emprendimiento agroecológico fracasadas}

Respecto al conocimiento que tienen los informantes sobre experiencias de producción ecológica que hayan fracasado, se observa que la proporción es ligeramente superior entre quienes sí conocen experiencias fracasadas (60\% de ellos) y quienes manifiestan no conocer ninguna (40\%).

De entre los entrevistados que manifiestan conocer otras experiencias que no han sobrevivido en el mercado de la producción agroecológica, podemos observar diferentes tipos de producción y, también, diversos motivos por los que dichas empresas han fracasado y desaparecido. Está claro en el discurso de E1: "Conozco una que hacían derivados de la miel. Su nombre era Agua de piedra o Piedra de agua, algo así; otra en maya que era de un grupo de mujeres que hacían mermeladas de Jamaica; otra que hacían mermeladas de habanero llamada Chacsinkin, en Petó, al sur de Yucatán. Los motivos de los fracasos de estas empresas, en la mayoría, fueron por dineroः se organizaban, producían y empezaban a bajar recursos y apoyos; cuando caían los apoyos financieros por parte del Gobierno mexicano era cuando empezaban los pleitos internos entre los integrantes".

La principal causa de los fracasos empresariales está relacionada con las propias características de la producción agroecológica o de carácter orgánico donde sus productos, comparados con los de la agricultura y ganaderías convencionales, son mucho más caros, al ser producciones más pequeñas, costosas y lentas, que no permiten un modelo de producción masiva y requieren más cuidados a lo largo de la cadena de producción. A esta causa se le une otra adicional, y es que se trata de pequeños microemprendedores locales que no disponen de un gran capital de partida para iniciar sus negocios, y hay que destacar que, tal y como se recoge de las aportaciones teóricas expuestas en páginas anteriores, existe, entre otras, una dimensión muy importante a la hora de apoyar y motivar estos emprendimientos, y es el hecho de que la transparencia y la confianza deben constituir dos elementos imprescindibles en los que se basen las relaciones de los microemprendedores con las Administraciones públicas para el fomento y consolidación de los emprendimientos (Sanagustín-Fons y Brunet-Icart, 2017; Ramírez et alii, 2017), aunque en la práctica no siempre se producen estos apoyos, bien por existir otras 
prioridades políticas o inadecuadas praxis de gestión que dificultan o impiden las estructuras necesarias para apoyar los emprendimientos.

También cabe destacar la falta de comercialización de estos productos por su escasa demanda debida a factores como el desconocimiento por parte de la población, la insuficiente consciencia y formación acerca de las bondades de los productos orgánicos $y$, como ya se ha argumentado en párrafos anteriores, la falta de cooperación entre los productores para el desarrollo de sus negocios.

\subsubsection{Razones para emprender en negocios agroecológicos}

Algo más de la mitad de los informantes (55\%) exponen que los motivos de emprender se centran en la existencia de una ideología naturalista que ha sido creada tras la reflexión o las experiencias vividas con el medio ambiente; pero también hay un número representativo que opina que los empresarios agroecológicos lo hacen porque está "de moda" y lo ven como una oportunidad para sus negocios (35\%). Así lo relata E1: "La mayoría de los contactos busco que sean por la ideología naturalista, sí, porque es por necesidad. Tuve una plática una vez con unos sabileros, y estaban divididos: unos querían seguir con lo orgánico tradicional y otros, con químicos que aceleraban el crecimiento, querían meter químicos, y ¿qué es lo que pasó?, que a los que no estaban casados con esta filosofía al rato les llegó la oportunidad de otro dinero, entonces alguien que lo hace por filosofía sigue viviendo así, es su forma de vida; estés o no estés..., seguirá con su filosofía“.

En Yucatán los emprendimientos se realizan en muchas ocasiones por ser contemplados como una oportunidad, pues el objetivo es vender a toda costa, $y$ no importan las implicaciones sociales, medioambientales y de salud que existen detrás de todo esto, con lo que el asesoramiento, la colaboración, la concienciación y la preservación de los productos locales quedan en segundo plano, siendo los jóvenes, más preparados en general, quienes asumen la iniciativa, como señala E6: "Bueno, hay una asociación en México que se ubica en Puebla que se llama Comida Lenta, está esa asociación y realmente esos dirigentes están en Italia. De todo lo que se recaba, una parte se va a la fundación que está en Italia, Bra (Italia), una parte se queda en la asociación, y la otra, al mercado. La finalidad es que desde su trinchera se sigan haciendo las actividades en pro del medio ambiente, cosa que aquí yo he tenido mucho problema, pues el mercado de la tierra en Yucatán es ir, vender y se acabó; no hay una colaboración o asesoramiento, etc. Es un mercado convencional, [... ] aquí la mentalidad final es la de los productores convencionales, que es vender y ya está..., el buscar mejorar, no“.

La paradoja es que no se predica con el ejemplo, pues muchos de estos emprendedores, a pesar de dedicarse a este tipo de emprendimientos, no son soste- 
nibles, no practican la alimentación saludable y otras prácticas que promueve la slow food. Están ahí, como explican algunos informantes, por la oportunidad que les ofrece este nuevo tipo de negocios donde cada vez hay una mayor demanda.

\subsubsection{Sector predominante en la producción orgánica}

Dentro de la producción agroecológica es el sector agrícola el que posee prioridad, dieciséis de los veinte entrevistados así lo afirman, argumentando que los principales productos que se cultivan y venden con calificativo orgánico son las frutas y las verduras, y algunas semillas también. (E3: "Hortalizas, te pongo el ejemplo..., las lechugas, que hay diferentes lechugas, diferentes hortalizas de hoja, frutas, digamos frutas de la época, y hay un producto que no está con nosotros, se menciona la sábila, pero la sábila te la menciono, que sí hay una producción, digamos que, mucho mayor de la sábila, pero se comercializa en mercados nacionales e internacionales, que es orgánica, porque, para que exportes, es el primer requisito").

Tan solo el 15\% de nuestros entrevistados se inclinan a favor de la ganadería como sector predominante, señalando el cerdo como ganado de producción ecológica predominante. Las opiniones al respecto en este sentido se verifican con el más de medio centenar de emprendedores que acuden semanalmente al Mercado Fresco (slow food) de Mérida, donde, ya sea de producción primaria o transformada, la mayoría de los productos que se pueden ver y comprar corresponden al sector agrícola, principalmente.

\section{Conclusiones}

El sector de la alimentación agroecológica es un sector en expansión que sigue generando empleo; cada vez con mayor frecuencia, se observa una tendencia entre jóvenes que quieren instalarse en el campo y desarrollar actividades vinculadas al mundo rural. El reto está en la profesionalización y en ser capaces de conciliar el saber hacer de los productores tradicionales con la nueva visión que pueden aportar esos jóvenes. La producción agroecológica se configura como un signo de distinción que ofrece mayores garantías de calidad para los consumidores, justificando así los postulados del movimiento internacional slow food.

El mercado ecológico está en crecimiento, tanto en México como a nivel mundial, y ofrece oportunidades que no tienen los productos convencionales. En Yucatán muchas familias viven en zonas rurales, por lo que apostar por alternativas para la mejora de la economía y la sostenibilidad se hace una tarea urgente. El emprender en el campo de la agroecología propicia beneficios en diversas vías, siendo uno de los más importantes la economía familiar, que deja de depender de la dinámica convencional. El movimiento slow food empieza a cobrar importancia 
en Yucatán desde hace una década aproximadamente. Existe una red social en la cual los productores y/o vendedores de los productos orgánicos interactúan e intercambian conocimientos y productos, al menos en el plano teórico; sin embargo, el grado de integración y cooperación social entre ellos es, sobre todo, bajo o medio. Lo que demuestra, por un lado, la existencia de, al menos, dos dimensiones perceptivas diferentes, la manifiesta, "existen redes sociales y de interacción", y la oculta, "su grado de penetración es escaso". Ello debido, principalmente, a que el perfil del yucateco es individualista, competidor e insolidario, y no se integra fácilmente. Por lo tanto, todos estos planteamientos se oponen claramente a la filosofía del movimiento slow food. Además, existen razones de índole sociocognitiva que evidencian las características territoriales. Se trata del desconocimiento mayoritario en relación con la existencia de cooperativas y asociaciones que permitan poder interrelacionarse. Asimismo, existe una confusión un tanto generalizada, en muchos casos, entre ambas figuras (cooperativa y asociación), lo cual confirma que este hecho propicie, aún más, esa falta de integración entre ellos.

La existencia de una ideología naturalista que ha sido creada tras la reflexión o las experiencias vividas con el medio ambiente y porque se trata de una moda concebida como una oportunidad para los negocios son las razones por las que los emprendedores mexicanos apuestan por el sector agroecológico. Siendo el sector que más presencia tiene en los emprendimientos el agrícola, con productos que se cultivan y venden con calificativo de orgánico, principalmente frutas, verduras y semillas, destaca también la presencia minoritaria de la ganadería, configurándose el ganado porcino como el producto rey en dicho sector.

En esta investigación se han analizado los motivos, razones y redes sociales establecidas por parte de los líderes de microemprendimientos agroecológicos; convendría continuar profundizando en cuestiones relevantes en este ámbito de estudio, como es la vinculación con el turismo gastronómico y experiencial, la cuestión de género, y cómo influye en un entorno de economía emergente, así como los apoyos o barreras institucionales que surgen ante dichos emprendimientos. 


\section{Referencias bibliográficas}

Ayuntamiento de Madrid (2017). "Curso Huerto urbano ecológico“. Disponible en: <https://www.madrid.es UnidadesDescentralizadas/Educacion_ Ambiental/ContenidosBasicos/Descriptivos/Huertos\%20urbanos\%20 comunitarios/Manual_huerto_urbano_ecológico/manual_huerto_urbano_ ecologico.pdf $>$.

Benito Hernández, Sonia (2009). “El papel de las microempresas en el desarrollo económico regional: las redes de cooperación empresarial en España". REVESCO, 99 (tercer cuatrimestre). ISSN: 1885-8031. Disponible en: $<$ http://webs.ucm.es/info/revesco/>.

Camacho Vera, Joaquín Huitzilihuitl; Cervantes Escoto, Fernando; Cesín Vargas, Alfredo y Palacios Rangel, María Isabel (2019). “Los alimentos artesanales y la modernidad alimentaria". Estudios sociales. Revista de alimentación contemporánea y desarrollo regional, 29 (53), 2-20. e19700. Disponible en: $<$ https://dx.doi.org/10.24836/es.v29i53.700>.

CÁrcamo, Héctor (2005)."Hermenéutica y análisis cualitativo," Cinta de Moebio. Revista de Epistemología de Ciencias Sociales, 23, 204-216.

Di Clemente, Elide; De Salvo, Paola y Hernández, José Manuel (2011). "Slow Tourism o turismo de la lentitud: un nuevo enfoque al desarrollo de territorios lentos". Book of Procedings, I. International Conference on Tourism E Managementes Studies. Algarve: 883-893.

Díaz Méndez, Cecilia y Gómez Benito, Cristóbal (coords.) (2008). Alimentación, consumo y salud. Barcelona: Ed. Fundación La Caixa. Colección Estudios Sociales, 24.

DíAz-Muñoz, José Guillermo (2015). Economías solidarias en América Latina. Guadalajara, Jalisco: ITESO.

Donovan Fortín, Patrick; Bravo, Gonzalo y González, Raúl (2010)."Microemprendimiento y desarrollo local en Chile: desafíos pendientes." Cuadernos del Cendes, 27 (73), 133-167.

FAO y OMS (2011). Codex Alimentarius. Programa Conjunto FAO/OMS sobre normas alimentarias. La Comisión de Codex Alimentarius. Disponible en: $<$ http://www.fao.org/tempref/codex/Publications/ProcManuals/Manual $\_20$ s.pdf $>$.

Gómez Cruz, Manuel Ángel (2005). “Red de consumidores de productos orgánicos en la UACH: Una experiencia de organización para el consumo". Revista Vinculando, 19 de enero de 2005.

Gómez Cruz, Manuel Ángel (2007). "Agricultura orgánica de México: situación, retos y tendencias 2005”. Revista Vinculando, 27 de julio de 2007. 
Gómez Bahillo, Carlos; Sanagustín-Fons, María Victoria y Sáez OliviTo, Enrique (2000). Población y recursos en Monegros: Análisis, diagnóstico y estrategias. Zaragoza: CEDDAR.

Gobierno de España (2009). EcoAgroCulturas. Proyecto de Fomento de la producción Agroecológica 2009-2011. Ecologistas en Acción, Fundación Biodiversidad, Emplea Verde 2007-2013 y la Unión Europea. Disponible en: $<$ https://www.empleaverde.es/sites/default/files/memoriasproyectos/ecologistas.pdf $>$.

Gobierno de España (2019). Red de emprendedores verde. Disponible en: <https://www.redemprendeverde.es>.

Guinjoan, Eloi; Badia, Anna y Tulla, Antoni F. (2016). "El nuevo paradigma de desarrollo rural. Reflexión teórica y conceptualización a partir de la rural web." Boletín de la asociación de Geógrafos Españoles, 17, 179-204. ISSN: 0212 9426. DOI: 10.2 1138/bage 2279.

Jones, Peter; Shears, Peter; Hillier, David; Comfort, Daphne y LowELL, Jonathan (2003). "Return to traditional values? A case study of Slow Food”. British Food Journal, 105 (4/5), 297-304. Disponible en: <https://doi. org/10.1108/00070700310477095>.

Junta de Extremadura et alii (2016). Plan Estratégico de apoyo al medio rural en Extremadura 2016-2020. Extremadura 2030. Disponible en: <http:// www.juntaex.es/filescms/con03/uploaded_files/SectoresTematicos/ DesarrolloRural/Diversificacion YDesarrolloRural/PlanEstrategico DeApoyoAlMedioRuralEnExtremadura.pdf $>$.

Leitch, Alison (2003). "Slow Food and the Politics of Pork Fat: Italian Food and European Identity”. Ethnos, 68 (4), 437-462. ISSN 0014-1844. DOI: $10.1080 / 0014184032000160514$.

Lupo, Eleonora (2012). "Slow Design: cultivar cultura y sensorialidad en la forma y en el uso de los artefactos." Temes de Disseny, 28, 44-55. Disponible en: <https://www.raco.cat/index.php/Temes/article/view/263096/350532>.

Marrone, Gianfranco (2011). "Brand on the runः mirada semiótica sobre Slow Food" Tópicos del Seminario, 26, 59-92.

Martínez-Quintana, Violante (2017). "El turismo de naturaleza: un producto turístico sostenible”. Arbor, 193 (785) a396, 1-14. DOI: <http*//dx.doi. org/10.3989/arbor.2017.785n3002>.

Martínez-Quintana, Violante (2019). Perspectiva sociológica turística en las sociedades del ocio. Madrid: Sanz y Torres.

Martínez-Quintana, Violante y Blanco-Gregory, Rocío (2013). "Hacia una gestión sostenible de las actividades turísticas en los espacios rurales y 
naturales". RIO. Revista Internacional de organizaciones, 10, 131-155. ISSN: 2013-570X. EISSN: 1886-4171.

Merçon, Juliana; Escalona Aguilar, Miguel Ángel; Noriega Armella, María Isabel; Figueroa Núñez, Ingrid Ivette; Atenko Sánchez, Aketzali y González Méndez, Enid Daniela (2012). "Cultivando la educación agroecológica. El huerto colectivo urbano como espacio educativo". RMIE, 17 (55), 1201-1224 (ISSN: 14056666).

Miele, Mara y Murdoch, Jonathan (2002). "The Practical Aesthetics of Traditional Cuisines: Slow Food in Tuscany”. Sociologia Ruralis, 42 (4), 312 328. DOI: 10.1111/1467-9523.00219.

Montaño Armendáriz, Angélica y Pérez Concha, Juan Carlos (2015). "Microemprendimiento de mujeres en los cabos bcs: una experiencia para el impulso del desarrollo local". En: XX Congreso Internacional de Contaduría, Administración e Informática. México, DF.

Murphy, Kevin; Croes, Robertico y Chen, Po-Ju (2012). "Turismo y agricultura. Un modelo de alimentación lenta para la promoción de productos agrícolas locales por medio del turismo en asistencia de los menos afortunados". Diálogos. Revista Electrónica de Historia, 13 (octubre, 2012), 65-82.

Naciones Unidas (2012). RIO+20. Conferencia de las Naciones Unidas. El futuro que queremos, Río de Janeiro, Brasil. Disponible en: < https://www. uncsd2012.org>.

OECD (2006). El nuevo paradigma rural. Politicas y Gobernanza. Madrid: Ministerio de Agricultura, Pesca y Alimentación. DOI: <https://dx.doi. org/10.1787/9788449107610-es>.

Petrini, Carlo (2007). Bueno, limpio y justo: principios de una nueva gastronomía. Madrid: Ed. Polifemo.

Pietrykowski, Bruce (2004). "You Are What You Eat: The Social Economy of the Slow Food Movement". Review of Social Economy, 62 (3), 307-321. DOI: 10.1080/0034676042000253927.

Puyal Español, Esther y Sanagustín-Fons, María Victoria (2006). "El liderazgo empresarial femenino: reflexiones y paradojas". Trabajo: Revista andalu$z a$ de relaciones laborales, 17, 169-186.

Ramírez Angulo, Natanael; Mungaray Lagarda, Alejandro; Aguilar Barceló, José Gabriel y Flores Anaya, Yadira Zulith (2017). "Microemprendimientos como instrumento de combate a la pobreza: una evaluación social para el caso mexicano". Innovar, 27 (64), 63-74.

Roiatti, Franca (2011). La rivoluzione della lattuga. Si può riscrivere l'economia del cibo? Prefazione di Carlo Petrini. Ed. Egea. 
Sanagustín-Fons, María Victoria (2011). Valores y ética empresarial. Un enfoque sociológico. Madrid: Trotta.

Sanagustín-Fons, María Victoria; Moseñe Fierro, José Antonio y Gómez y Patiño, María (2011). "Rural Tourism. A sustainable alternative”. Applied Energy, 88 (22), 551-557. DOI: 10.1016/j.apenergy+2010.08.031.

Sanagustín-Fons, María Victoria y Brunet-Icart, Ignasi (2017). "Modelo de innovación socio-institucional en la administración pública”. RIO. Revista Internacional de Organizaciones, 19, 93-116.

Sanagustín-Fons, María Victoria; Lafita-Cortés, Teresa y Moseñe, José Antonio (2018). "Social Perception of Rural Tourism Impact: A Case Study". Sustainability, 2 (10), 339. DOI: 10.3390/su10020339-

Schwentesius Rintermann, Rita (2010). "Producción orgánica y mercados locales en México". Revista Vinculando. Disponible en: <http://vinculando.org/organicos/produccion_organica_y_mercados_locales_en_mexico. html>.

Stow Food (2015). "Nuestra historia. Slow Food: la historia de una idea”. Disponible en: <https://www.slowfood.com/es/quienes-somos/nuestra-histo$\mathrm{ria} />$.

Slow Food (2017). "México tiene su segundo Mercado de la Tierra". Disponible en: <https //www.slowfood.com/es/mexico-segundo-mercado-la-tierra/>.

Viqueira, Carmen y Palerm, Ángel (1954). "Alcoholismo, brujería y homicidio en dos comunidades rurales de México". América indígena, XIV (1), 7-36.

Vilaplana i Batalla, Montse (2011). Cultura alimentaria. Del fast food al Slow Food. Revista Offarm, 30 (6), 53-56.

Zamilpa, Johanan; Schwentesius Rindermann, Rita y Ayala Ortiz, Daniel Ariel (2016). "Estado de la cuestión sobre las críticas a la agricultura orgánica”. Acta Univ, 26 (2), 20-29. ISSN 2007-9621. Disponible en: <http:// dx.doi.org/10.15174/au.2016.854>. 


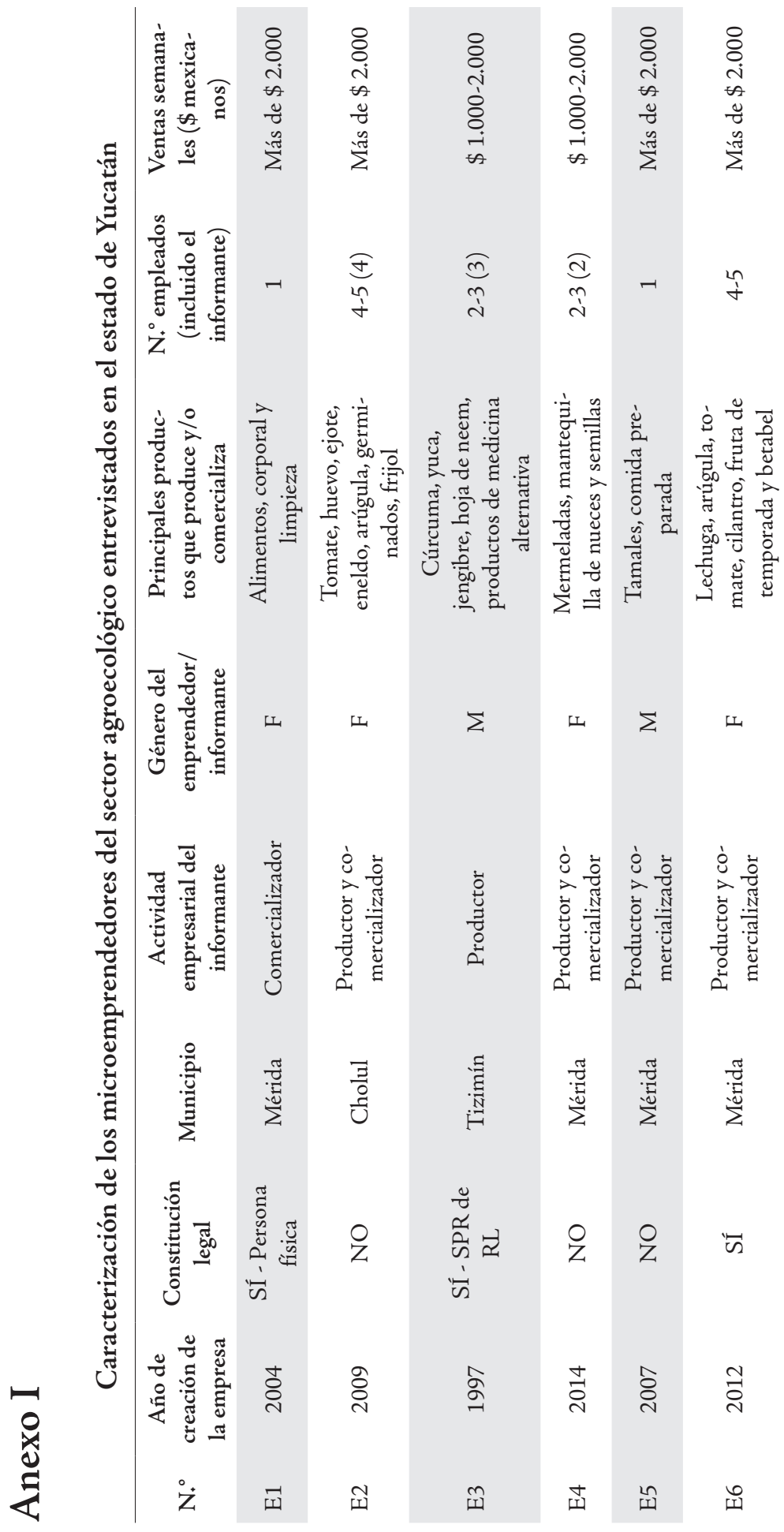




\begin{tabular}{|c|c|c|c|c|c|c|}
\hline 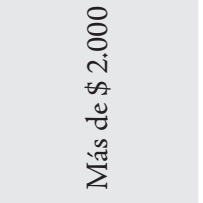 & $\begin{array}{l}8 \\
8 \\
i \\
\infty \\
0 \\
0 \\
0 \\
i \\
i\end{array}$ & 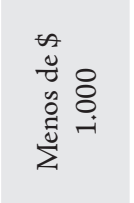 & $\begin{array}{l}8 \\
\delta \\
i \\
0 \\
0 \\
0 \\
0 \\
i \\
i\end{array}$ & 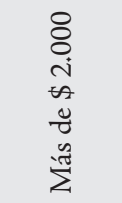 & 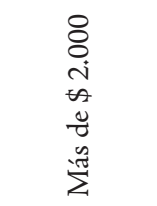 & $\begin{array}{l}8 \\
8 \\
i \\
i \\
0 \\
0 \\
i \\
i\end{array}$ \\
\hline$\hat{n}$ & $\hat{i}$ & $\hat{i}$ & $\hat{n}$ & $\hat{i}$ & $\begin{array}{l}\infty \\
i n \\
0 \\
0 \\
i n \\
\sum_{i}^{\infty}\end{array}$ & 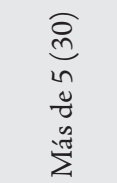 \\
\hline 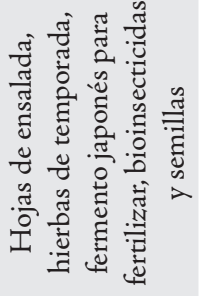 & 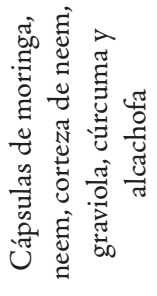 & 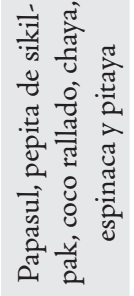 & 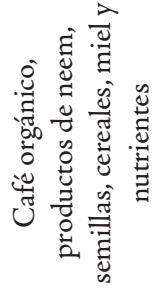 & 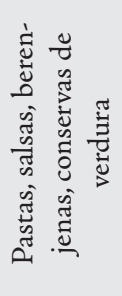 & 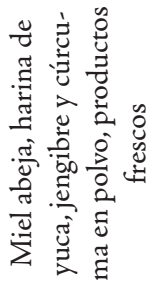 & 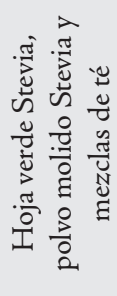 \\
\hline 山 & $\Sigma$ & $\Sigma$ & 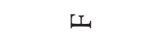 & 山 & 山 & $\Sigma$ \\
\hline 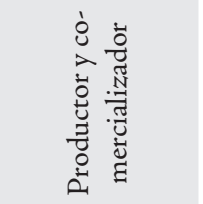 & 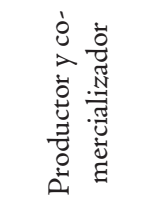 & 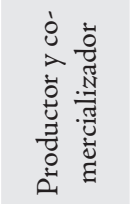 & 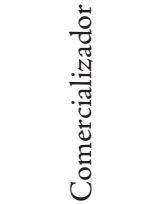 & 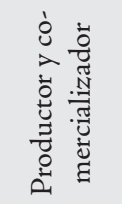 & 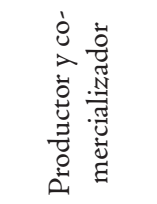 & 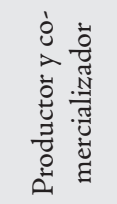 \\
\hline 总 & 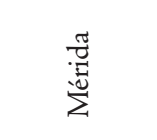 & 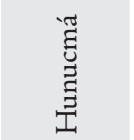 & 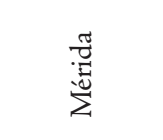 & 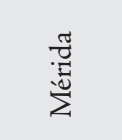 & 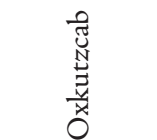 & $\begin{array}{l}\frac{\pi}{D^{*}} \\
\sum_{\Sigma}^{\pi}\end{array}$ \\
\hline $\begin{array}{l}\text { ○ } \\
\text { Z }\end{array}$ & 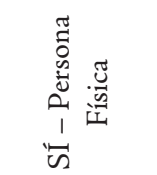 & $\begin{array}{l}O \\
Z\end{array}$ & " & $" \sqrt{ }$ & $\sqrt{n}$ & 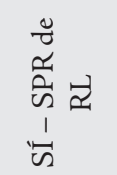 \\
\hline 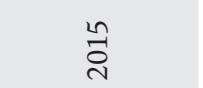 & $\underset{⿱ 丷}{\stackrel{\nabla}{d}}$ & $\frac{n}{\tilde{N}}$ & ڤิ & $\vec{\sim}$ & ন্ & $\stackrel{\sim}{\stackrel{乛}{\sim}}$ \\
\hline 玒 & $\begin{array}{l}\infty \\
\text { II }\end{array}$ & ్ి & 利 & $\overrightarrow{\bar{I}}$ & $\stackrel{\sim}{-1}$ & $\stackrel{m}{\underline{m}}$ \\
\hline
\end{tabular}


Rocío Blanco-Gregory, M. ${ }^{a}$ Violante Martínez-Quintana, M. ${ }^{a}$ Victoria Sanagustín-Forns

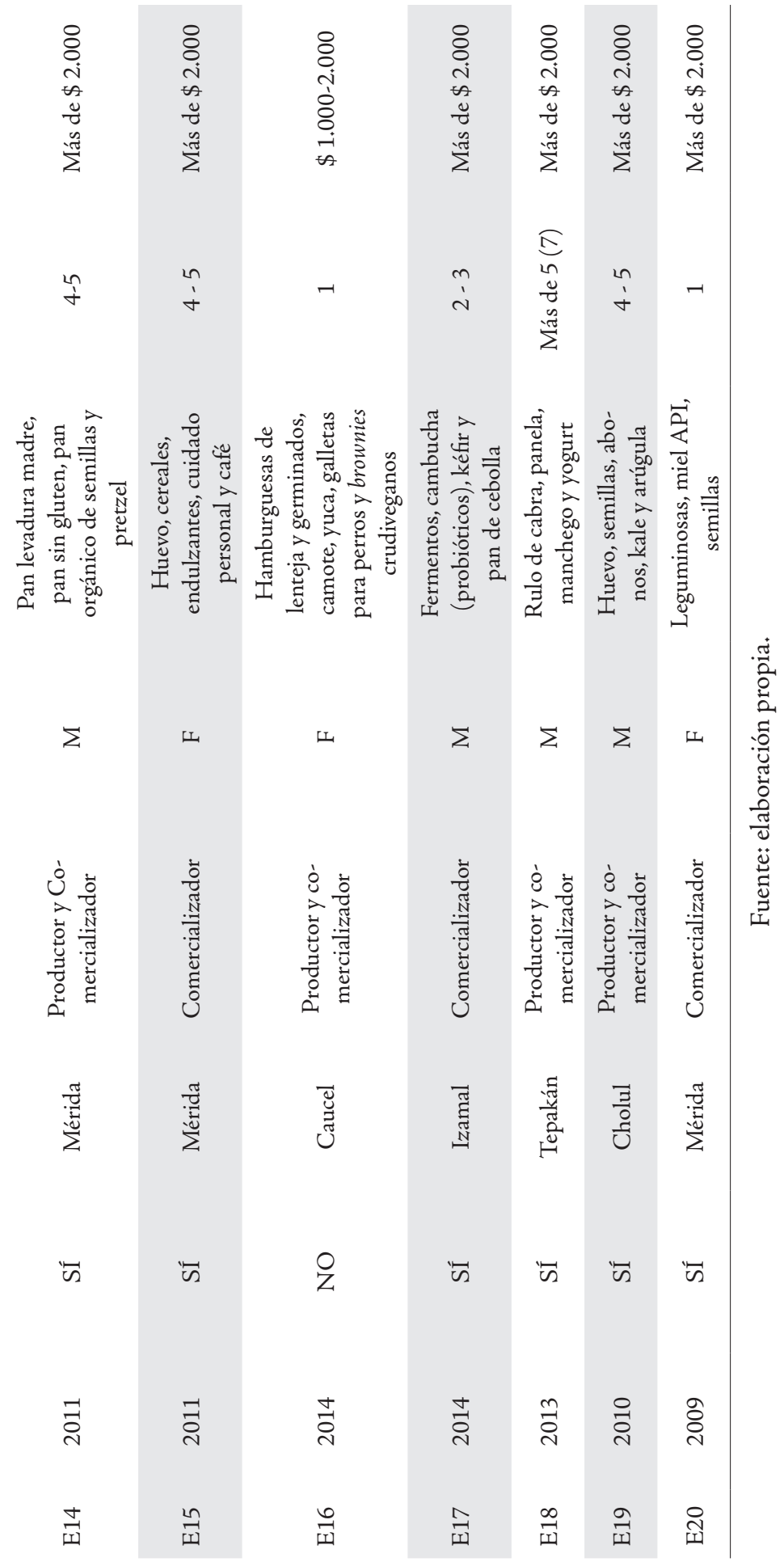

